\title{
Exploring the Role and Mechanism of Imatinib in Chronic Unpredictable Mild Stress-induced Depression Model of Rats
}

\author{
Xiaohui Wang1, Yajing Li ${ }^{2}$, Yinxue Shen ${ }^{3}$, Dongzi Zhang ${ }^{4 *}$ \\ ${ }^{1}$ Wenzhou Medical University, Major in Clinical Medicine, Wenzhou, Zhejiang, CHINA. \\ ${ }^{2}$ Department of General, Community Health Service Center (District Hospital), Baoding, Hebei, CHINA. \\ ${ }^{3}$ School of Applied Psychology, North China University of Technology, Tangshan, Hebei, CHINA. \\ ${ }^{4}$ Psychological Counseling Clinic, Affiliated Hospital of Hebei University, Baoding, Hebei, CHINA.
}

\begin{abstract}
Aim: The study was planned to examine the potential effect of imatinib in chronic unpredictable mild stress (CUMS)-induced depression model of rats. Materials and Methods: Male rats were subjected to the 6-week CUMS with unpredictable stressors to induce depression and the imatinib was started from the $4^{\text {th }}$ week (for 21 days during 6 weeks of the protocol). Results: CUMS significantly increased immobility time and decreased consumption of sucrose in the swimming and sucrose preference test respectively. Furthermore, a considerable reduction in the Brain-derived neurotrophic factor (BDNF) levels and an increase in phosphorylated NF-kB levels (p-NF-kB) were noticed in the prefrontal cortex (PFC). However, imatinib $(25$ and $50 \mathrm{mg} / \mathrm{kg}$ ) significantly reversed sucrose consumption as well as reduced immobility times in CUMS-subjected rats, suggesting the antidepressant potential of imatinib. Moreover, imatinib treatment significantly increases in the levels of BDNF and a decrease in the p-NF-kB levels in the PFC of the brain of stress-subjected rats. The co-administration of tropomyosin receptor kinase B (TrkB) antagonist, ANA-12 $(0.25$ and $0.5 \mathrm{mg} / \mathrm{kg})$ with imatinib $(50 \mathrm{mg} / \mathrm{kg})$ considerably attenuated imatinib-mediated antidepressant effects in sucrose preference behavior and immobility period in CUMS subjected rats. ANA-12 abolished imatinibinduced effects in stress-subjected rats. Conclusion: The antidepressant actions of imatinib may be attributed to an increase in the BDNF levels and a decrease in the p-NF$k B$ levels in the stress-susceptible PFC region of the CUMS subjected rats.
\end{abstract}

Key words: CUMS, Depression, p-NF-kB, BDNF, Imatinib.

\section{INTRODUCTION}

Depression is a recognized as a serious psychological disorder to affects $20 \%$ of the population. Worldwide and remains one of the important factors leading to disability. ${ }^{1}$ The characteristic features of depression in humans include persistently sad mood, decreased pleasure in day to day activities, reduced concentration, inability to take decisions, helplessness, loss of interest and persistent thoughts of committing suicides. ${ }^{1,2}$ These symptoms significantly affect the quality of life. $^{3}$ Several antidepressant drugs have been employed for the management of depressive symptoms; however, more than $40 \%$ of patients do not achieve satisfactory results in response to existing antidepressant drugs. ${ }^{1,4}$ Therefore, it is the need to investigate new and effective therapeutic agents that may effectively manage the symptoms of depression. CUMS is a widely used model and it mimics the clinical conditions of human depression., ${ }^{5,6}$ CUMS model is preferred over other models because the unpredictability of stressor application significantly reduces the induction of stress adaptation.
Submission Date: 04-01-2020; Revision Date: 27-04-2020; Accepted Date: 19-05-2020

DOI: 10.5530/ijper.54.3.118 Correspondence: Dr. Dongzi Zhang

Psychological Counseling Clinic, Affiliated Hospital of Hebei University, Baoding-071000, Hebei, CHINA.

Phone: +86-531-18803123320

E-mail: zhangdongzi6688@ sina.com

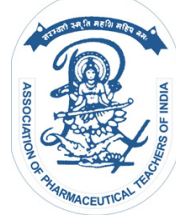

www.ijper.org 
Imatinib (tyrosine kinase inhibitor) is used clinically in the different type of cancers. ${ }^{7,8}$ Moreover, preclinical studies have demonstrated its beneficial effects in different neuronal diseases such as Alzheimer's disease, spinal cord injury, stroke, etc. ${ }^{9,10}$ Studies have also documented that tyrosine kinase activation modulates the anxious and depressed behavior. ${ }^{11,12}$ Moreover, it has also been reported that imatinib may affects the behavior of cancer patients. In line with this, evidence has shown that discontinuation of tyrosine kinase inhibitors including imatinib leads to the anxiety or depression in chronic myeloid leukemia patients. ${ }^{13}$ However, there have not been direct studies delineating the potential of imatinib in depression and related behavioral disorders. Brain-derived neurotrophic factor (BDNF) performs diverse biological functions in the brain via activation of tropomyosin receptor kinase B (TrkB). The BDNF receptors are found in abundance in stress-responsive prefrontal cortex region. ${ }^{14,15}$ Interestingly, it has been found that signaling via BDNF and TrkB plays a major role in the depression pathophysiology and $\operatorname{TrkB}$ inhibitors are commonly employed as pharmacological tools to find out the role of BDNF in different research studies. ${ }^{16,17}$ Several lines of evidence have shown the decrease in BDNF-TrkB signaling in the stress responsive hippocampus and prefrontal cortex in different models of depression including unpredictable mild stress, ${ }^{18}$ chronic social defeat stress ${ }^{19,20}$ and learned helplessness. ${ }^{21}$ Furthermore, BDNF has shown a protective role in depression treatment in preclinical studies. ${ }^{22,23}$

Nuclear factor-kappa B (NF-kB) regulates many physiological processes in the body. ${ }^{24,25}$ It has also been indicated that stress activates the $\mathrm{p}-\mathrm{NF}-\mathrm{kB}$ signaling in the brain to induce behavioral changes. ${ }^{26,27}$ Several lines of preclinical evidence have shown that $\mathrm{p}-\mathrm{NF}-\mathrm{kB}$ is up regulated in depression and it has also been suggested that an increase in $\mathrm{p}-\mathrm{NF}-\mathrm{kB}$ may possibly contribute to the depression. ${ }^{28}$ It has also been demonstrated that NF-kB inhibitor attenuates the harmful effects of stress, anxiety, post-traumatic stress disorder and depression. ${ }^{28-30}$ Examining the key role of BDNF/TrkB signaling and $\mathrm{p}-\mathrm{NF}-\mathrm{kB}$ in the depression along with behavior modulating potential of imatinib, the study was designed to (a) explore the therapeutic potential of imatinib in attenuating depression in stress-subjected rats and (b) explore the possible contribution of $\mathrm{BDNF}$ and $\mathrm{p}-\mathrm{NF}-\mathrm{kB}$ in imatinib-mediated antidepressant action in a depressed rats.

\section{MATERIALS AND METHODS}

\section{Animals, Chemicals and Drugs}

Male Wistar albino rats (200-250 g) were kept in the maintained laboratory conditions $\left(25^{\circ}-26^{\circ} \mathrm{C}\right.$ and natural day and night cycle). The animals-related experiments were approved by the Wenzhou Medical University (ethical number, WZH20190828). Imatinib mesylate was procured from Dalian Meilun Biotech Co., Ltd (Dalian, China). ANA-12 (Maybridge, UK) was dissolved in DMSO. The estimation of BDNF was done by using Promega ELISA kits and p-NF-kB levels were assessed

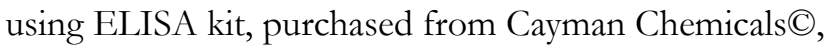
Tallin, Estonia.

\section{Employment of stress to induce depression}

In this study, animals were given six-week chronic unpredictable mild stress (42 days) to induce depression. The animals were subjected to eight different types of stressors randomly. The different stressors included paired caging for $48 \mathrm{hr}$ in which rat were placed in new cages with unknown rats; 10 min level shaking; 1 min tail pinching; placing the rats in a wet bedding for 24 $\mathrm{hr}, 24 \mathrm{hr}$ cage tilting, $5 \mathrm{~min}$ swimming in the cold water at temperature $13 \pm 1^{\circ} \mathrm{C}$ or hot water at temperature $40 \pm 1{ }^{\circ} \mathrm{C}$, reversal of light and dark cycle and food deprivation for about $24 \mathrm{hr}^{31-33}$

\section{Forced swim stress Test (FST)}

This swimming test is the commonly employed tests to assess depression in animals. In this test, rats were put in the water-filled glass cylinders of length $40 \mathrm{~cm}$ and diameter $18 \mathrm{~cm}$. During the four minutes test, the immobility period i.e. the time when the rat is unable to maintain swimming and starts to float instead of swimming in water-filled glass cylinders was noted. ${ }^{34,35}$

\section{Sucrose preference test}

On the $42^{\text {nd }}$ day, this test was conducted to assess the extent of anhedonia (not able to experience pleasure), which is a major characteristic feature of depression. The volume of sucrose solution consumed by an animal was employed as an indicator of anhedonia and it is significantly reduced in animals experiencing anhedonia. Sucrose is a pleasurable feeling for rodents and rodents fail to experience the pleasure of drinking sweet water during the depression. It is in contrast to normal rats that prefer to drink sweet water in comparison to normal water if given a choice. The rats were given access to a bottle full of $1 \%$ sucrose solution and another bottle full of normal water. After $1 \mathrm{hr}$, the consumption of sucrose solution and normal water was assessed by 
estimating the fluid remaining in those bottles. The total intake of sucrose solution was calculated in percentage to total water intake. ${ }^{35-37}$

\section{Determination of BDNF and p-NF-kB}

After completion of behavioral testing, the prefrontal cortex region was collected from the brain and was homogenized in the lysis buffer which also contains protease and phosphatase inhibitors. With addition of these protease and phosphatase inhibitors, the degradation of the extracted proteins can be prevented and the best protein yield and activity can be achieved. The homogenate was subjected to centrifugation $(10000 \times \mathrm{g})$ for $10 \mathrm{~min}$ at $4^{\circ} \mathrm{C}$. Thereafter, the supernatants were removed and used for ELISA-based estimation of BDNF and p-NF-kB levels (Elfving et al. 2010; Bali and Jaggi, 2016). The procedure for ELISA was followed as per the instructions of the manufacturer. The biochemical results were expressed as nanograms/ $\mathrm{mg}$ of protein. In the homogenate, the levels of protein were quantified using the Folin-Lowery method.

\section{Protocol}

Seven groups with eight rats per group were employed.

\section{Group I: Normal Control}

The animals were placed in a cage for six weeks and were not disturbed except during the cleaning of cages.

\section{Group II: Chronic unpredictable stress}

Rats were given six week chronic unpredictable stress as described above. On $42^{\text {nd }}$ day, the behavioral testing was done and thereafter, the rats were sacrificed to measure the BDNF as well as p-NF-kB levels in the brain.

Groups III and IV: Imatinib (25 and $50 \mathrm{mg} / \mathrm{kg}$ ) in chronic unpredictable stress

Imatinib (25 mg/kg) in group III and imatinib (50 mg/ $\mathrm{kg}$ ) in group IV was administered for the last 21 days (starting from $4^{\text {th }}$ week) to stress-subjected animals.

Groups V and VI: ANA-12, TrkB antagonist (0.25 and $0.5 \mathrm{mg} / \mathrm{kg}$ ) and imatinib (50 mg/kg) in chronic unpredictable stress

BDNF inhibitor i.e. ANA-12 $(0.25 \mathrm{mg} / \mathrm{kg})$ in group V and ANA-12 $(0.5 \mathrm{mg} / \mathrm{kg})$ in group VI was co-administered with imatinib for 21 days.

Group VII: DMSO and imatinib $(50 \mathrm{mg} / \mathrm{kg})$ in chronic unpredictable stress

DMSO (solvent of ANA-12) was co-administered with imatinib for 21 days.
In all groups, behavioral testing was performed on the $42^{\text {nd }}$ day. Afterward, the rats were sacrificed for estimation of BDNF and p-NF-kB levels in the brain.

\section{Statistical Analysis}

Graph Pad Prism was used for statistical analysis of the data obtained from present research. One-way ANOVA followed by post hoc Tukey's test was employed for comparing the statistical differences among different groups. The results were expressed as mean \pm S.D. $p<0.05$ was considered as statistically significant.

\section{RESULTS}

\section{Effects of stress on the behavioral as well as biochemical parameters}

Exposure to six week of unpredictable stress led to significant behavioral alterations, assessed on $42^{\text {nd }}$ day i.e. at the end of the protocol. A significant decrease in sucrose consumption was also noticed in the sucrose preference test on $42^{\text {nd }}$ day in comparison to the normal group, suggesting the significant development of anhedonia (Figure 1). Further, an increase in the immobility was also noted as compared to normal rats in a forced swim test (Figure 2). Moreover, a significant decrease in the BDNF and an increase in $\mathrm{p}-\mathrm{NF}-\mathrm{kB}$ levels were observed in CUMS-subjected rats, assessed on the $42^{\text {nd }}$ day of the protocol (Figures 3 and 4).

\section{Effects of imatinib on behavioral alterations in the sucrose consumption and swim stress in unpredictable stress-subjected rats}

Treatment of chronic unpredictable stress subjected rats with imatinib (25 and $50 \mathrm{mg} / \mathrm{kg}$ ) for three weeks exhibited a significant increase in the sucrose consumption in comparison to the stress control (Figure 1). Moreover, there was a decrease in the immobility time on $42^{\text {nd }}$ day in imatinib-treated rats in comparison to the stress control rats in a swim test (Figure 2). The effects of imatinib at dose $50 \mathrm{mg} / \mathrm{kg}$ were significantly higher than the $25 \mathrm{mg} / \mathrm{kg}$ imatinib. Three weeks of imatinib treatment led to a significant increase in BDNF and a decrease in $\mathrm{p}-\mathrm{NF}-\mathrm{kB}$ levels in stress-subjected rats (Figures 3 and 4).

\section{Effects of ANA-12 on imatinib-mediated behavioral as well as biochemical alterations in unpredictable stress- subjected rats}

In this study, co-administration of ANA-12 (0.25 and $0.5 \mathrm{mg} / \mathrm{kg}$ ) with imatinib (50 mg/kg) for three weeks significantly abolished imatinib-mediated restoration of sucrose preference behavior and immobility period in chronic unpredictable mild stress-subjected rats 
(Figure 1). There was a marked decrease in the sucrose consumption and increase in the immobility period on $42^{\text {nd }}$ day in sucrose preference and forced swim tests, respectively in response to co-administration of ANA-12 and imatinib in chronic unpredictable stresssubjected rats (Figure 2). The effects of ANA-12 at 0.5 $\mathrm{mg} / \mathrm{kg}$ were significantly higher than observed at the low dose i.e. $0.25 \mathrm{mg} / \mathrm{kg}$. Co-administration of TrkB antagonist abolished the imatinib-mediated increase in BDNF and decrease in the $\mathrm{p}-\mathrm{NF}-\mathrm{kB}$ levels in chronic unpredictable stress-subjected rats (Figures 3 and 4).

\section{DISCUSSION}

In the present research, exposure of rats to six week chronic unpredictable mild stress i.e. 42 days led to significant behavioral alterations in terms of decrease

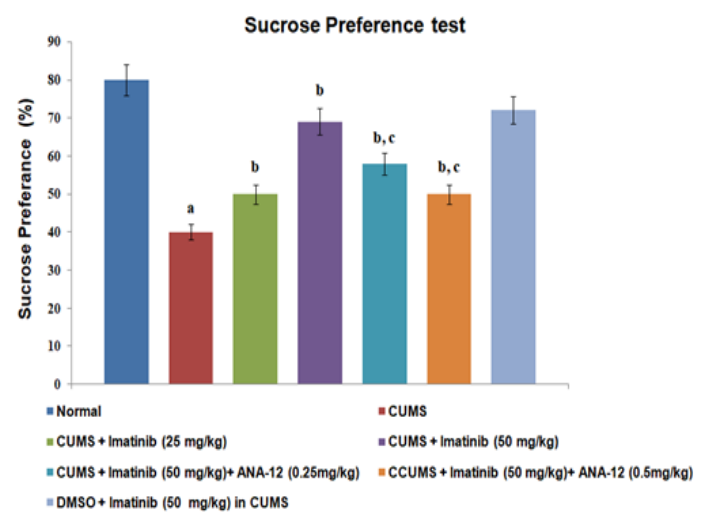

Figure 1: Effects of chronic unpredictable mild stress and pharmacological interventions on the sucrose consumption in sucrose preference test: Values are expressed as mean \pm S.D. with $n=8$ a $p<0.05$ as compared to non-stress control; ${ }^{b} p<0.05$ as compared to CUMS control; ${ }^{c} p<0.05$ as compared to imatinib in CUMS.

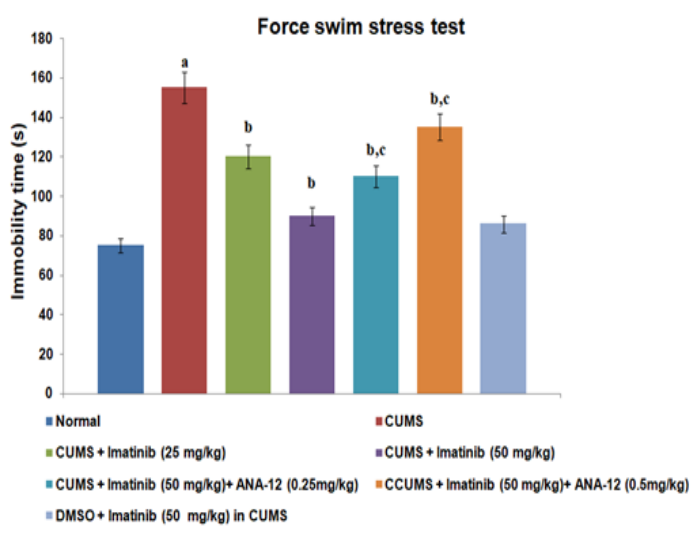

Figure 2: Effects of chronic unpredictable mild stress and pharmacological interventions on the immobility period in force swim stress test: Values are expressed as mean \pm S.D. with $n=8$ a $p<0.05$ as compared to non-stress control; ${ }^{b} p<$ 0.05 as compared to CUMS control; ${ }^{c} p<0.05$ as compared to imatinib in CUMS. in the consumption of sucrose and increase in the immobility in the sucrose preference and the forced swim stress tests, respectively. The chronic unpredictable stress model is a widely adopted model among the preclinical depression models to screen the antidepressant agents. The duration and methodology of this stress model was based on the published literature. ${ }^{16,18,38}$ In this model, animals are exposed to different stressors in a random and unpredictable manner to induce the symptoms of depression. ${ }^{36}$ This model offers the advantage to other models as no stress adaptation is observed in this model. The sucrose preference test is a common behavioral test to assess the development of anhedonia in rats and mice. ${ }^{31,32}$ The sucrose preference test is based on the assessment of preference of rat for a sweet solution than normal water. The lack of preference for sucrose

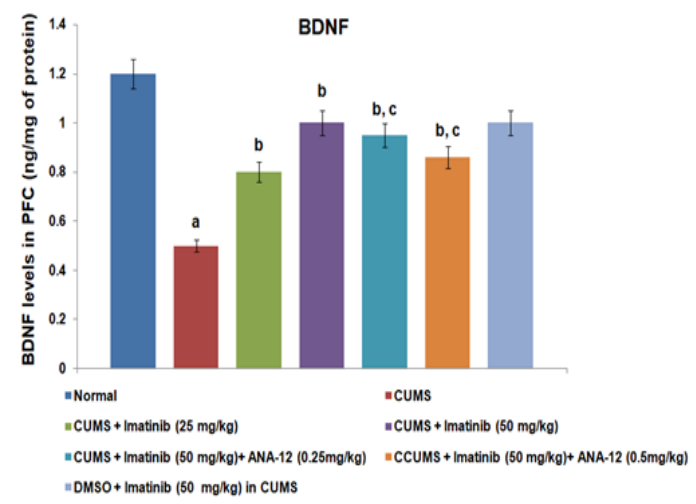

Figure 3: Effects of chronic unpredictable mild stress and pharmacological interventions on the BDNF levels in prefrontal cortex: Values are expressed as mean \pm S.D. with $n=8$ in each group a $p<0.05$ as compared to non-stress control; ${ }^{b} p<$ 0.05 as compared to CUMS control; ${ }^{c} p<0.05$ as compared to imatinib in CUMS.

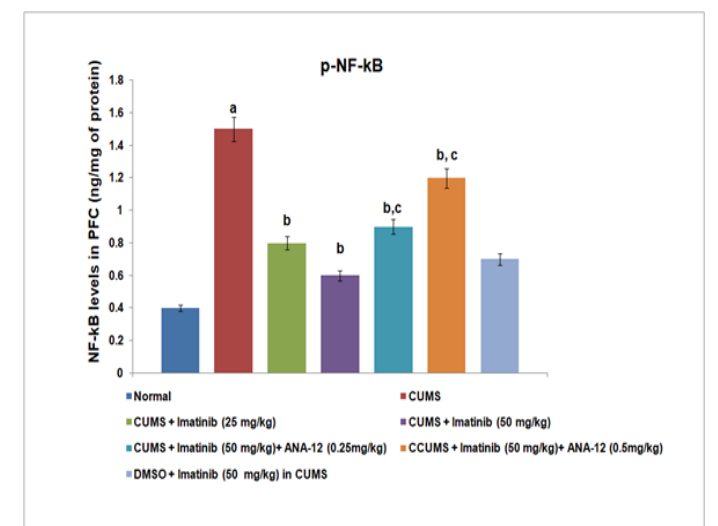

Figure 4: Effects of chronic unpredictable mild stress and pharmacological interventions on the p-NF-kB levels in the prefrontal cortex: Values are expressed as mean \pm S.D. with $n=8 .{ }^{a} p<0.05$ as compared to non-stress control; ${ }^{b} p<0.05$ as compared to CUMS control; ' $p<0.05$ as compared to imatinib in CUMS. 
shows a decrease in hedonic activity, therefore this test is commonly used as an indicator of anhedonia-like behaviors. ${ }^{39}$ In other words, it indicates the existence of depressive behavior, suggesting the development of depression in stress-subjected rats. Similarly, an increase in the immobility period i.e. presence of hopelessness in the swim stress test is also commonly employed to assess the existence of depressive behavior in animals. ${ }^{35}$ Therefore, the absence of preference of sucrose and increased immobility in the present experiment, suggests the significant development of depression like behavior in response to chronic unpredictable stress.

Three week imatinib (25 and $50 \mathrm{mg} / \mathrm{kg}$ ) treatment i.e. for 21 days significantly ameliorated CUMS-induced depression-like behavior in terms of attenuation of decreased sucrose preference and increased immobility as compared to the stress control. A marked increase in preference for sucrose and decrease in the immobility period on $42^{\text {nd }}$ day in imatinib-treated rats was observed as compared to the stress control, suggesting the antidepressant potential of imatinib. Imatinib is clinically employed for the treatment of cancers. ${ }^{7,8}$ On the basis of previously published literature ${ }^{40}$ the doses of imatinib were selected. However, several studies have shown the therapeutic potential of imatinib in the brain disorders. ${ }^{9}{ }^{10}$ There have also been studies showing that tyrosine kinases may modulate the depression like behavior. ${ }^{11,12}$ However, it is the first study demonstrating the anti-depressant action of imatinib in unpredictable stress-subjected animals.

To explore the possible mechanisms of imatinibmediated antidepressant actions, the levels of BDNF and $\mathrm{p}-\mathrm{NF}-\mathrm{kB}$ were assessed in unpredictable stresssubjected rats. A significant decrease in the BDNF levels and an increase in the p-NF-kB levels were observed in the PFC in stress-subjected rats on the $42^{\text {nd }}$ day of protocol. BDNF is a neurotrophin and several studies have shown the downregulation of BDNF in the brain in depression. ${ }^{41,42}$ Furthermore, its role has also been reported in antidepressant treatment as BDNF infusion shown to significantly attenuate the depressive behavior in animal model of depression. ${ }^{22,23,43}$ Furthermore, studies have shown the upregulation of nuclear transcription factor, $\mathrm{p}-\mathrm{NF}-\mathrm{kB}$ in different models of depression and it has been postulated that increased $\mathrm{p}-\mathrm{NF}-\mathrm{kB}$ can contribute to the development of depression. ${ }^{28,44}$ Interestingly, a reciprocal relationship has been found between $\mathrm{p}-\mathrm{NF}-x \mathrm{~B}$ and BDNF in the physiopathology of depression. ${ }^{17,28,44}$ Accordingly, it may be proposed that a decrease in the BDNF and an increase in the $\mathrm{p}-\mathrm{NF}-\mathrm{kB}$ following exposure to stress may significantly contribute to the depressive-behavior in rats.

In this experimental study, three week imatinib treatment significantly increased the BDNF levels and a decrease $\mathrm{p}-\mathrm{NF}-\mathrm{kB}$ levels in the PFC of stress-subjected rats. It suggests that imatinib-mediated antidepressant actions may be secondary to an increase in the BDNF levels and a decrease in the $\mathrm{p}-\mathrm{NF}-\mathrm{kB}$ levels. In other words, it may be possible to suggest that imatinib modulates BDNF/ $\mathrm{p}-\mathrm{NF}-\mathrm{kB}$ signaling to produce antidepressant effects in chronic unpredictable stress-subjected rats. There have been earlier studies showing the potential of imatinib in attenuating the expression of $\mathrm{p}-\mathrm{NF}-\mathrm{kB}$ in different conditions supporting the results of our present study. ${ }^{45}$ However this is the first study to delineate the increase in the levels of BDNF in the stress-sensitive prefrontal cortex of the brain following long term treatment with imatinib.

To further explore the relationship between imatinib and BDNF, ANA-12 i.e. TRkB antagonist was co-administered with imatinib in stress-subjected animals. The co-administration of ANA-12 TrkB antagonist $(0.25$ and $0.5 \mathrm{mg} / \mathrm{kg})$ along with imatinib $(50 \mathrm{mg} / \mathrm{kg}$ ) for three weeks considerably attenuated imatinib-mediated restoration of sucrose preference behavior and immobility period in chronic unpredictable stress-subjected rats. The doses of ANA-12 were also selected on the basis of previously published literature. ${ }^{46}$ A marked decrease in the preference for sucrose and increase in the immobility was observed on $42^{\text {nd }}$ day in response to ANA-12 and imatinib treatment in stresssubjected rats. BDNF produces diverse biological actions through the activation of $\operatorname{TrkB} \cdot{ }^{47}$ Accordingly, it can be proposed that the $\operatorname{TrkB}$ antagonist-mediated decrease in the antidepressant effects of imatinib is secondary to the blocked actions of BDNF. In other words, it may be proposed that imatinib might increases BDNF levels, which in turn activates TrkB to produce antidepressant actions.

In the present study, co-administration of $\operatorname{TrkB}$ antagonists significantly abolished imatinib-mediated decrease in the $\mathrm{p}-\mathrm{NF}-\mathrm{kB}$ as well as an increase in BDNF levels in chronic unpredictable stress subjected rats. It suggests that the TrkB antagonist blocks the actions of the imatinib by decreasing the BDNF and increasing $\mathrm{p}-\mathrm{NF}-\mathrm{kB}$ levels. A significant increase in the p-NF-kB levels in the presence of TrkB inhibitor suggests that $\mathrm{BDNF}$ has a negative influence on the expression of $\mathrm{p}-\mathrm{NF}-\mathrm{kB}$ and hence, the blockade of BDNF actions may lead to increase in the $\mathrm{p}-\mathrm{NF}-\mathrm{kB}$ levels, which may be manifested in the form of an increase in depressive behavior. 


\section{CONCLUSION}

Imatinib significantly attenuated the depression like behavior in stress-induced depression in rats. This antidepressive potential of imatinib may be due to an increase in the levels of BDNF and a decrease in the $\mathrm{p}-\mathrm{NF}-\mathrm{kB}$ in the prefrontal cortex region. In other words, imatinib-mediated modulation of $\mathrm{BDNF} / \mathrm{NF}-\mathrm{kB}$ signaling may contribute to attenuating depressivesymptoms in unpredictable stress-subjected rats.

\section{ACKNOWLEDGEMENT}

The authors are grateful for the support and funding by Department of Psychological Counseling Clinic, Affiliated Hospital of Hebei University, Baoding, Hebei, 071000, China

\section{CONFLICT OF INTEREST}

The authors have no conflict of interesting regarding the study.

\section{ABBREVIATIONS}

CUMS: Chronic Unpredictable Mild Stress; BDNF: Brain derived neurotrophic factor; p-NF-kB: Phosphorylated nuclear factor $\mathrm{kB}$ levels; PFC: Prefrontal cortex; 5TrkB: Tropomyosin receptor kinase B; DMSO: Dimethyl sulfoxide; FST: Forced swim stress Test.

\section{REFERENCES}

1. Smith K. Mental health: A world of depression. Nature. 2014;515(7526):181.

2. Zhang FF, Peng W, Sweeney JA, Jia ZY, Gong QY. Brain structure alterations in depression: Psychoradiological evidence. CNS Neurosci Ther. 2018;24(11):994-1003.

3. Sivertsen H, Bjørkløf GH, Engedal K, Selbæk G, Helvik AS. Depression and Quality of Life in Older Persons: A Review. Dement Geriatr Cogn Disord. 2015;40(5-6):311-39.

4. Thomas SJ, Shin M, Mclnnis MG, Bostwick JR. Combination therapy with monoamine oxidase inhibitors and other antidepressants or stimulants: Strategies for the management of treatment-resistant depression. Pharmacotherapy. 2015;35(4):433-49.

5. Zhu S, Shi R, Wang J, Wang JF, Li XM. Unpredictable chronic mild stress not chronic restraint stress induces depressive behaviours in mice. Neuroreport. 2014;25(14):1151-5.

6. Liu WX, Wang J, Xie ZM, Xu N, Zhang GF, Jia M, et al. Regulation of glutamate transporter 1 via BDNF-TrkB signaling plays a role in the antiapoptotic and antidepressant effects of ketamine in chronic unpredictable stress model of depression. Psychopharmacology. 2016;233(3):405-15.

7. Baccarani M, Saglio G, Goldman J, Hochhaus A, Simonsson B, Appelbaum $\mathrm{F}$, et al. European Leukemia Net. Evolving concepts in the management of chronic myeloid leukemia: Recommendations from an expert panel on behalf of the European Leukemia Net. Blood. 2006;108(6):1809-20.

8. Ben AE, Demetri GD. A safety evaluation of imatinib mesylate in the treatment of gastrointestinal stromal tumor. Expert Opin Drug Saf. 2016;15(4):571-8.
9. Gagało I, Rusiecka I, Kocić I. Tyrosine Kinase Inhibitor as a new Therapy for Ischemic Stroke and other Neurologic Diseases: Is there any Hope for a Better Outcome?. Curr Neuropharmacol. 2015;13(6):836-44.

10. Kumar M, Kulshrestha R, Singh N, Jaggi AS. Expanding spectrum of anticancer drug, imatinib, in the disorders affecting brain and spinal cord. Pharmacol Res. 2019;143:86-96.

11. Boxall AR, Lancaster B, Garthwaite J. Tyrosine kinase is required for longterm depression in the cerebellum. Neuron. 1996;16(4):805-13.

12. Kutlu MG, Cole RD, Connor DA, Natwora B, Gould TJ. Tyrosine receptor kinase $B$ receptor activation reverses the impairing effects of acute nicotine on contextual fear extinction. J Psychopharmacol. 2018;32(3):367-72.

13. Sogawa R, Kimura S, Yakabe R, Mizokami Y, Tasaki M, Sueoka-Aragane $\mathrm{N}$, et al. Anxiety and depression associated with tyrosine kinase inhibitor discontinuation in patients with chronic myeloid leukemia. Int J Clin Oncol. 2018;23(5):974-9.

14. Aoki C, Wu K, Elste A, Len Gw, Lin Sy, McAuliffe G, et al. Localization of brainderived neurotrophic factor and TrkB receptors to postsynaptic densities of adult rat cerebral cortex. J Neurosci Res. 2000;59(3):454-63.

15. Kaplan DR, Miller FD. Neurotrophin Signal Transduction in the Nervous System. Curr Opin Neurobiol. 2000;10(3):381-91.

16. Zhang JC, Wu J, Fujita Y, Yao W, Ren Q, Yang C, et al. Antidepressant effects of TrkB ligands on depression-like behavior and dendritic changes in mice after inflammation. Int J Neuropsychopharmacol. 2014;18(4).

17. Caviedes A, Lafourcade C, Soto C, Wyneken U. BDNF/NF-kB Signaling in the Neurobiology of Depression. Curr Pharm Des. 2017;23(21):3154-63.

18. Liu WX, Wang J, Xie ZM, Xu N, Zhang GF, Jia M, et al. Regulation of glutamate transporter 1 via BDNF-TrkB signaling plays a role in the antiapoptotic and antidepressant effects of ketamine in chronic unpredictable stress model of depression. Psychopharmacology. 2016;233(3):405-15.

19. Ma M, Ren Q, Yang C, Zhang JC, Yao W, Dong C, et al. Adjunctive treatment of brexpiprazole with fluoxetine shows a rapid antidepressant effect in social defeat stress model: Role of BDNF-TrkB signaling. Sci Rep. 2016;6:39209

20. Dong C, Zhang JC, Yao W, Ren Q, Ma M, Yang C, et al. Rapid and sustained antidepressant action of the mGlu2/3 receptor antagonist MGS0039 in the social defeat stress model: Comparison with ketamine. Int J Neuropsychopharmacol. 2017;20(3):228-36.

21. Yang B, Yang C, Ren Q, Zhang JC, Chen QX, Shirayama Y, et al. Regional differences in the expression of brain-derived neurotrophic factor (BDNF) pro-peptide, proBDNF and preproBDNF in the brain confer stress resilience. Eur Arch Psychiatry Clin Neurosci. 2016;266(8):765-9.

22. Bawari S, Tewari D, Argüelles S, Sah AN, Fazel NS, Xu S, et al. Targeting BDNF signaling by natural products: Novel synaptic repair therapeutics for neurodegeneration and behavior disorders. Pharmacol Res. 2019;104458.

23. Nasrallah P, Haidar EA, Stephan JS, ElHayek L, Karnib N, Khalifeh M, et al. Branched-chain amino acids mediate resilience to chronic social defeat stress by activating BDNF/TRKB signaling. Neurobiol Stress. 2019;11:100170

24. Piva R, Belardo G, Santoro MG. NF-kappa B: A stress-regulated switch for cell survival. Antioxid Redox Signal. 2006;8(3-4):478-86.

25. Koo JW, Russo SJ, Ferguson D, Nestler EJ, Duman RS. Nuclear factorkappa $B$ is a critical mediator of stress-impaired neurogenesis and depressive behavior. Proc Natl Acad Sci U S A. 2010;107(6):2669-74.

26. Madrigal JL, Hurtado O, Moro MA, Lizasoain I, Lorenzo P, Castrillo A, et al. The increase in TNF-alpha levels is implicated in NF-kappaB activation and inducible nitric oxide synthase expression in brain cortex after immobilization stress. Neuropsychopharmacology. 2002;26:155-63.

27. Madrigal JL, Moro MA, Lizasoain I, Lorenzo P, Castrillo A, Boscá L, et al. Inducible nitric oxide synthase expression in brain cortex after acute restraint stress is regulated by nuclear factor kappaB-mediated mechanisms. J Neurochem. 2001;76(2):532-8.

28. Chang D, Zhao J, Zhang X, Lian H, Du X, Yuan R, et al. Effect of ketamine combined with DHA on lipopolysaccharide-induced depression-like behavior in rats. Int Immunopharmacol. 2019;75:105788

29. Manchanda RK, Jaggi AS, Singh N. Ameliorative potential of sodium cromoglycate and diethyldithiocarbamic acid in restraint stress-induced behavioral alterations in rats. Pharmacol Rep. 2011;63(1):54-63.

30. Bierhaus A, Wolf J, Andrassy M, Rohleder N, Humpert PM, Petrov D, et al. A mechanism converting psychosocial stress into mononuclear cell activation. Proc Natl Acad Sci USA. 2013;100(4):1920-5. 
31. Willner P, Towell A, Sampson D, Sophokleous S, Muscat R. Reduction of sucrose preference by chronic unpredictable mild stress and its restoration by a tricyclic antidepressant. Psychopharmacology. 1987;93(3):358-64.

32. Willner P. Validity, reliability and utility of the chronic mild stress model of depression: a 10-year review and evaluation. Psychopharmacology. 1997;134(4):319-29.

33. Willner P. Chronic mild stress (CMS) revisited: Consistency and behaviouralneurobiological concordance in the effects of CMS. Neuropsychobiology. 2005;52(2):90-110.

34. Detke MJ, Rickels M, Lucki I. Active behaviors in the rat forced swimming test differentially produced by serotonergic and noradrenergic antidepressants. Psychopharmacology. 1995;121(1):66-72.

35. Huang HJ, Zhu XC, Han QQ, Wang YL, Yue N, Wang J, et al. Ghrelin alleviates anxiety and depression-like behaviors induced by chronic unpredictable mild stress in rodents. Behav Brain Res. 2017;326:33-43.

36. Papp M, Moryl E, Willner P. Pharmacological validation of the chronic mild stress model of depression. Eur J Pharmacol. 1996;296(2):129-36.

37. Xing H, Zhang K, Zhang R, Shi H, Bi K, Chen X. Antidepressant-like effect of the water extract of the fixed combination of Gardenia jasminoides, Citrus aurantium and Magnolia officinalis in a rat model of chronic unpredictable mild stress. Phytomedicine. 2015;22(13):1178-85.

38. Zhu X, Xia O, Han W, Shao M, Jing L, Fan Q, et al. San Improves DepressiveLike Behavior in Rats through Modulation of $\beta$-Arrestin 2-Mediated Pathways in Hippocampus. Evid Based Complement Alternat Med. 2014;902516.

39. Wiborg O. Chronic mild stress for modeling anhedonia. Cell Tissue Res. 2013;354(1):155-69.
40. Zhou L, Sun X, Huang Z, Zhou T, Zhu X, Liu Y, et al. Imatinib Ameliorated Retinal Neovascularization by Suppressing PDGFR- $\alpha$ and PDGFR- $\beta$. Cell Physiol Biochem. 2018;48(1):263-73.

41. Karege F, Perret G, Bondolfi G, Schwald M, Bertschy G, Aubry JM. Decreased serum brain-derived neurotrophic factor levels in major depressed patients. Psychiatry Research. 2002;109(2):143-8.

42. Huang TL, Lee CT, Liu YL. Serum brain-derived neurotrophic factor levels in patients with major depression: Effects of antidepressants. Journal of Psychiatric Research 2008;42(7):521-5.

43. Shirayama Y, Chen ACH, Nakagawa S, Russell DS, Duman RS. Brainderived neuro-trophic factor produces antidepressant effects in behavioral models of depression. The Journal of Neuroscience: The Official Journal of the Society for Neuroscience. 2002;22(8):3251-61.

44. Pesarico AP, Sartori G, Brüning CA, Mantovani AC, Duarte T, Zeni G, et al. A novel isoquinoline compound abolishes chronic unpredictable mild stressinduced depressive-like behavior in mice. Behav Brain Res. 2016;307:73-83.

45. Ciarcia R, Vitiello MT, Galdiero M, Pacilio C, lovane V, D'Angelo D, et al. Imatinib treatment inhibit IL-6, IL-8, NF-KB and AP-1 production and modulate intracellular calcium in CML patients. J Cell Physiol. 2012;227(6):2798-803.

46. Alzarea S, Rahman S. Effects of alpha-7 nicotinic allosteric modulator PNU 120596 on depressive-like behavior after lipopolysaccharide administration in mice. Prog Neuropsychopharmacol Biol Psychiatry. 2018;86:218-28. doi: 10.1016/j.pnpbp.2018.05.018. Epub 2018 May 22

47. Li Z, Zhang Y, Tong Y, Tong J, Thiele CJ. Trk inhibitor attenuates the BDNF/ TrkB-induced protection of neuroblastoma cells from etoposide in vitro and in vivo. Cancer Biol Ther. 2015;16(3):477-83.

\section{PICTORIAL ABSTRACT}

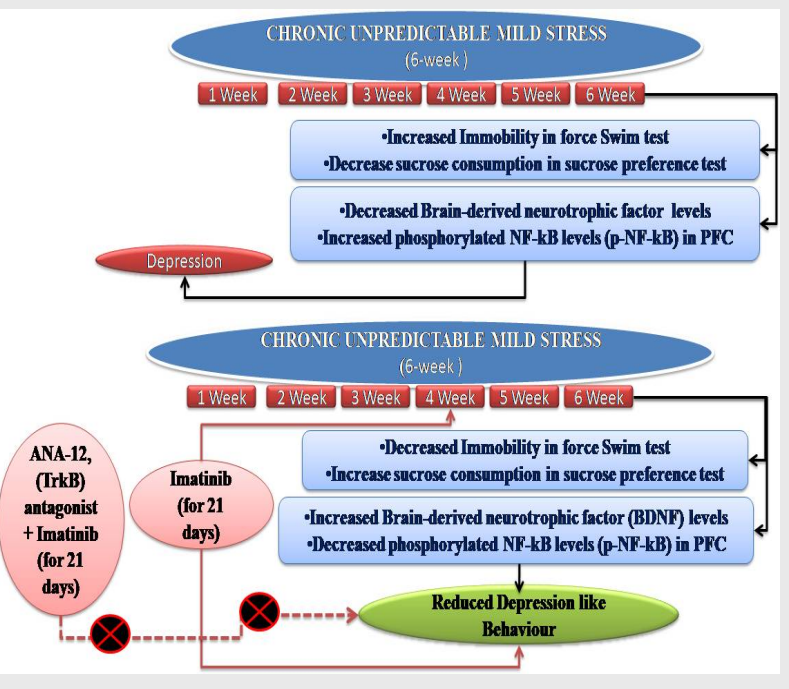

\section{SUMMARY}

The research was conducted to examine the role of imatinib in stress-induced depression in rats. CUMS significantly increased immobility time and decrease consumption of sucrose along with a noteworthy decline in the Brain-derived neurotrophic factor (BDNF) levels and increase in phosphorylated $\mathrm{NF}-\mathrm{kB}$ levels $(\mathrm{p}-\mathrm{NF}-\mathrm{kB})$ in the prefrontal cortex (PFC) region of brain. However, imatinib treatment (25 and $50 \mathrm{mg} / \mathrm{kg}$ ) significantly reversed sucrose consumption as well as reduced immobility times in CUMS-subjected rats. Moreover, imatinib attenuates the levels of BDNF and p-NF-kB in brain of stresssubjected rats. Co-administration of ANA-12 (0.25 and $0.5 \mathrm{mg} / \mathrm{kg}$ ) with imatinib $(50 \mathrm{mg} / \mathrm{kg}$ ) considerably attenuated imatinib-mediated antidepressant effects in sucrose preference behavior and immobility period in CUMS subjected rats. ANA-12 abolished imatinibmediated decrease in the $\mathrm{p}-\mathrm{NF}-\mathrm{kB}$ levels and an increase in BDNF levels in stress-subjected rats. Imatinib-mediated modulation of $\mathrm{BDNF} / \mathrm{NF}-\mathrm{kB}$ signaling may contribute to attenuate depression-like behavior in unpredictable stress-subjected rats. 


\section{About Authors}

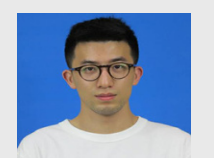

Xiaohui Wang, Wenzhou Medical University, Major in Clinical Medicine, Wenzhou, Zhejiang, 325035, China.

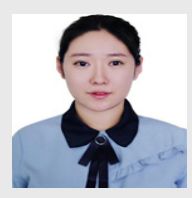

Yixue Shen, Applied Psychology School of Psychology North China University of Technology, Tangshan, Hebei, 063000, China.

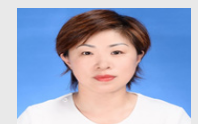

Yajing Li, Department of General, Community Health Service Center (District Hospital), Baoding, Hebei Province, 071000, China.

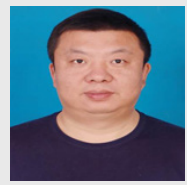

Dongzi Zhang, Psychological Counseling clinic, Affiliated Hospital of Hebei University, Baoding, Hebei, 071000, China.

Cite this article: Wang $\mathrm{X}, \mathrm{Li} \mathrm{Y}$, Shen $\mathrm{Y}$, Zhang D. Exploring the Role and Mechanism of Imatinib in Chronic Unpredictable Mild Stress-induced Depression Model of Rats. Indian J of Pharmaceutical Education and Research. 2020;54(3):682-9. 